\title{
Diagnostic implication of fibrin degradation products and D-dimer in aortic dissection-author's reply
}

\author{
Shuangshuang Li ${ }^{1 *}$, Jian Dong ${ }^{1 *}$, Lei Zhang ${ }^{1 *}$, Zhiqing Zhao ${ }^{2}$, Rui Feng ${ }^{1}$, Qingsheng Lu ${ }^{1}$, Jian Zhou $^{1}$, \\ Junmin Bao ${ }^{1}$, Zaiping Jing ${ }^{1}$ \\ ${ }^{1}$ Department of Vascular Surgery, ${ }^{2}$ Department of Surgery, Changhai Hospital, Second Military Medical University, Shanghai 200433, China \\ *These authors contributed equally to this work. \\ Correspondence to: Zaiping Jing, MD; Jian Zhou, MD; Junmin Bao, MD. Department of Vascular Surgery, Changhai Hospital, Second Military \\ Medical University, Shanghai 200433, China. Email: xueguanky@163.com; zhoujian1-2@163.com; baojm@xueguan.net. \\ Provenance: This is an invited article commissioned by the Section Editor Lei Zhang (Department of Vascular Surgery, Changhai Hospital, Second \\ Military Medical University, Shanghai, China). \\ Response to: Luo CY, Roan JN. Re-visiting D-dimers and fibrin degradation products for the diagnosis of acute aortic dissection. J Thorac Dis \\ 2017;9:1744-7.
}

Akutsu K. What are diagnostic implications and limitations of assessing D-dimer and fibrin degradation products levels in the management of patients with acute aortic dissection? J Thorac Dis 2017;9:2214-6.

Submitted Aug 14, 2017. Accepted for publication Aug 31, 2017.

doi: $10.21037 /$ jtd.2017.09.06

View this article at: http://dx.doi.org/10.21037/jtd.2017.09.06

Acute aortic dissection (AAD) is a life-threatening disease requiring an early diagnosis and rapid treatment to prevent death. The annual incidence of $\mathrm{AAD}$ was about three cases per 100,000 , with a high mortality rate of $24.4 \%$ for type A and $10.7 \%$ for type B without urgent treatment (1). About $20 \%$ $\mathrm{AAD}$ patients were with non-specific symptoms, and up to $40 \%$ of patients were undiagnosed until necropsy (2). Given the atypical presentations and time dependent mortality, sensitive and quicker predictive diagnosis methods are paramount to take first-aids measures.

Even though imaging diagnosis, such as computed tomography angiography (CTA), transesophageal echocardiography (TEE), or magnetic resonance angiography (MRA) were still recognized as the gold standard for AAD diagnosis, the disadvantages of them were obvious and inevitable too (3). Invasive and expensive imaging examinations were not the first-line choices, causing an inevitable and further logistical delay for treatment. Besides, the use of these methods was based on typical symptoms, doctor's experience and high index of clinical suspicious. As a result, patients without atypical symptoms may be mis-diagnosed or delayed diagnosis, because they have little probability to take imaging examination. During the past decades, many studies focused on the use of D-dimer in AAD diagnosis. What's more, the AD Task Force of the European Society of Cardiology recommended D-dimer measurement as part of the routine initial diagnostic steps (4).

Based on our previous study, the research about diagnostic value of AAD using D-dimer and fibrin degradation products (FDP) was published in recent issue of Scientific reports. We really appreciated that Dr. Akutsu and Luo put forward their insightful comments on our article. The diagnosis function of D-dimer had been widely explored in previous studies with a high sensitivity, while researches on the diagnosis value of FDP were relatively scarce $(5,6)$. As we know, both FDP and D-dimer were generated by the fibrinolytic system. Nagaoka $e t$ al. found a positive linear correlation between the plasma levels of D-dimer and FDP (7). We believed that a similar diagnosis tendency or complementary advantage would exist between the two biomarkers. In our study, the diagnosis value seemed not high with a sensitivity of $68.8 \%$ for D-dimer and $53.1 \%$ for FDP. This fact somewhat may evoke suspicious in the clinical utility for AAD diagnosis. As pointed out by Akutsu and Luo, several factors perhaps affected the value of the 
two indicators. It should be highlighted that patients in subacute phase or without specific symptom were included in this study and their D-dimer and FDP were tested at first visit. Besides, all of the included patients were with thrombosis false lumen, an average age of 55.32 years and $72 \%$ male. As reported, AAD patients with younger age, subacute phase and male sex may show a lower D-dimer or FDP value (8). As to false lumen condition, there had controversy result. For FDP, Hagiwara et al. reported a significantly higher level in patients with patent false lumen than thrombosed, while opposite result was showed in study of Nagaoka et al. (6,7). The lower value of D-dimer and FDP may be caused by the combined effect of those factors. For that matter, we didn't preset a cut-off value and the result would be more applicable. Besides, this article took focus on the diagnosis value of D-dimer and FDP for AAD not to explore the influence factors, then further studies are needed to explore the influence factors of D-dimer and FDP in detail.

As recommended in the two comments, the D-dimer should be used in patients who were unlikely or with low probability to be suffering from AAD. However, it should be also used with caution even D-dimer at a normal range, as there is no sufficient definite evidence in favor of the diagnosis role of D-dimer or FDP (9). Even a low sensitivity of biomarkers may be helpful in raising the index of $\mathrm{AD}$ clinical suspicion and directing patients towards imaging.

In summary, early and effective diagnosis method of $\mathrm{AAD}$ is imperative, and biomarkers would show the wide application prospect, especially in suspicious $\mathrm{AD}$ patients. Taking into account the limitation and immaturity of using an isolated biomarker, we suggest to combine FDP and $\mathrm{D}$-dimer in clinic application for AAD. Further prospective multi-center studies were necessary to determine the certain role or characteristics of D-dimer and FDP that are used alone or in combination in $\mathrm{AD}$ diagnostic algorithm.

\section{Acknowledgements}

Funding: This work was supported by the National Natural Science Foundation of China (No. 81370441), and Shanghai Health System Diseases Important Joint Research Project
(No. 2014ZYJB0401).

\section{Footnote}

Conflicts of Interest: The authors have no conflicts of interest to declare.

\section{References}

1. Pape LA, Awais M, Woznicki EM, et al. Presentation, Diagnosis, and Outcomes of Acute Aortic Dissection: 17Year Trends From the International Registry of Acute Aortic Dissection. J Am Coll Cardiol 2015;66:350-8.

2. Olin JW, Fuster V. Acute aortic dissection: the need for rapid, accurate, and readily available diagnostic strategies. Arterioscler Thromb Vasc Biol 2003;23:1721-3.

3. Baliga RR, Nienaber CA, Bossone E, et al. The role of imaging in aortic dissection and related syndromes. JACC Cardiovasc Imaging 2014;7:406-24.

4. Erbel R, Alfonso F, Boileau C, et al. Diagnosis and management of aortic dissection. Eur Heart J 2001;22:1642-81.

5. Shao N, Xia S, Wang J, et al. The role of D-dimers in the diagnosis of acute aortic dissection. Mol Biol Rep 2014;41:6397-403.

6. Hagiwara A, Shimbo T, Kimira A, et al. Using fibrin degradation products level to facilitate diagnostic evaluation of potential acute aortic dissection. J Thromb Thrombolysis 2013;35:15-22.

7. Nagaoka K, Sadamatsu K, Yamawaki T, et al. Fibrinogen/ fibrin degradation products in acute aortic dissection. Intern Med 2010;49:1943-7.

8. Hazui $\mathrm{H}$, Nishimoto $M$, Hoshiga $M$, et al. oung adult patients with short dissection length and thrombosed false lumen without ulcer-like projections are liable to have false-negative results of D-dimer testing for acute aortic dissection based on a study of 113 cases. Circ J 2006;70:1598-601.

9. Mussa FF, Horton JD, Moridzadeh R, et al. Acute Aortic Dissection and Intramural Hematoma: A Systematic Review. JAMA 2016;316:754-63.
Cite this article as: Li S, Dong J, Zhang L, Zhao Z, Feng R, Lu Q, Zhou J, Bao J, Jing Z. Diagnostic implication of fibrin degradation products and D-dimer in aortic dissection-author's reply. J Thorac Dis 2017;9(10):E941-E942. doi: 10.21037/jtd.2017.09.06 